\title{
Quantitative Measurement of Retinal Vessel Density in Non- proliferative and Proliferative Macular Telangiectasia Type 2 with Optical Coherence Tomography Angiography
}

\author{
(D) Ali Demircan, (D) Zeynep Alkın, (D) Ceren Yeşilkaya, (D) Gülşah Gümüş, (D) Dilek Yaşa, (D) Gürkan Erdoğan \\ Istanbul Beyoğlu Eye Training and Research Hospital, Clinic of Retina, Istanbul, Turkey
}

\section{Abstract}

Objective: The purpose of the study was to quantify the optical coherence tomography angiography (OCTA) features of non-proliferative and proliferative macular telangiectasia type 2 (MacTel2). The retinal vessel density (RVD) of the superficial and deep layers of the retina were studied.

Methods: This cross-sectional and observational study was carried out at İstanbul Beyoğlu Eye Training and Research Hospital. Patients clinically diagnosed with MacTel2 underwent fundus photography, fluorescein angiography, spectral domain OCT, and OCTA imaging. Superficial and deep RVD in the foveal and parafoveal regions were calculated using device automated software.

Results: Thirteen eyes of 8 patients ( 4 male, 4 female) with a mean age of $64.6 \pm 3.8$ years were included. The mean RVD of the superficial fovea and parafovea were $30.3 \% \pm 9.3 \%$ and $49.5 \% \pm 6.4 \%$ in non-proliferative MacTel2, compared with $39.4 \% \pm 3.3 \%$ and $43.8 \% \pm 2.9 \%$ in the proliferative MacTel2, respectively. No statistically significant difference was found between non-proliferative and proliferative MacTel2 $(p=0.31, p=0.41$; respectively). The mean deep foveal and parafoveal RVD was $30.4 \% \pm 7.8 \%$ and $50.7 \% \pm 3.8 \%$ in non-proliferative MacTel2 versus $47.5 \% \pm 0.2 \%$ and $55.3 \% \pm 8.4 \%$ in proliferative MacTel2, respectively. There was a significant difference in deep foveal RVD between proliferative and non-proliferative MacTel2 patients, whereas no difference was found in deep parafoveal RVD ( $p=0.02$ and $p=0.23$, respectively).

Conclusion: The mean deep foveal RVD was significantly higher in proliferative MacTel2 than in non-proliferative MacTel2. Measurement of RVD in the deep retinal layers by OCTA may have diagnostic value in patients with proliferative MacTel2.

Keywords: Macular telangiectasia type 2, optical coherence tomography angiography, non-proliferative, proliferative

\section{INTRODUCTION}

Type 2 macular telangiectasia (MacTel2) is a progressive, bilateral retinal vascular disease that arises within the temporal, juxtafoveal region of the macula. Initially, the microvascular features of non-proliferative MacTel2 include telangiectatic abnormalities within the deep retinal capillary plexus (DRCP), temporal to the fovea, and these changes continue to invade the superficial retinal capillary plexus with subsequent extension circumferentially to involve the perifoveal microvasculature $(1,2)$.
In the later proliferative stages of MacTel2, neovascularization may arise and extend under the retina, often leading to pigment deposition and disciform scar formation (3). Fluorescein angiography (FA) continues to be the gold standard method for confirming the diagnosis of proliferative MacTel2 $(1,2)$. Gass and Oyakawa (4) have reported that vascular alterations mainly include the DRCP and that the late leakage of fluorescence on FA seems to arise from the outer retina in non-proliferative MacTel2. Diffuse hyperfluorescence conceals the morphological changes 
in the vascular network, and FA is insufficient to give detailed information about the deep retinal layers and pathological changes therein (5). On the other hand, FA is an invasive imaging method, and it may not be possible to repeat the test at each visit.

Optical coherence tomography angiography (OCTA) is a novel, non-invasive imaging method that provides high-resolution imaging of retinal morphology, primarily individual vascular layers, as an important advantage (6,7). Additionally, OCTA allows better visualization of subretinal neovascularization (SNV), compared with FA, due to the absence of fluorescence leakage (8).

In this study, we aimed to determine whether retinal vascular density (RVD) was different between non-proliferative and proliferative MacTel2. With this new, non-invasive technique, we aim to identify proliferative changes in MacTel2 without the need for FA.

\section{METHODS}

Patients diagnosed with MacTel 2 at İstanbul Beyoğlu Eye Training and Research Hospital were included in this retrospective, crosssectional, and observational study. Institutional review board approval was achieved from İstanbul Beyoğlu Eye Training and Research Hospital Review Board (approval date: 31.08.2018, approval no: 18/I-1). The study was carried out in accordance with the principles of the Declaration of Helsinki. As a criterion for inclusion in the study, patients had to be diagnosed with non-proliferative or proliferative MacTel2 in at least one eye. Patients with retinal vein occlusion, diabetic retinopathy, choroidal neovascularization secondary to age-related macular degeneration, previous vitreoretinal surgery history, or history of photodynamic therapy were excluded from the study. All patients underwent a complete ophthalmologic evaluation, including slit lamp biomicroscopy, intraocular pressure measurement with applanation tonometry, and dilated fundus examination after best corrected visual acuity measurement using Snellen charts. Besides a comprehensive ocular examination, all patients underwent color fundus imaging, FA imaging with HRA 2 (Germany, Heidelberg, Heidelberg Engineering), and spectral domain OCT imaging (Germany, Heidelberg, Heidelberg Engineering). In addition, patients were imaged with the Optovue AngioVue system (Optovue, CA, Inc., Freemont) for OCTA imaging. This device has a scanning speed of 70,000 A-scans per second using a light source with a bandwidth of $50 \mathrm{~nm}$ and centered at $840 \mathrm{~nm}$. The retinal region of a volume scan included a $3 \mathrm{~mm} \times 3 \mathrm{~mm}$ field of view obtained with at least 49 B-scans per volume. Patients whose images had inadequate signal strength were excluded from the study. A split-spectrum amplitude-decor correlation angiography algorithm was used to calculate a flow map for each scan. In this study, two-layer imaging of the OCT angiogram was analyzed, including superficial and DRCs. In this study, we included two layers from the en face OCT angiogram in our analysis: The superficial retinal capillaries and the DRCS. The OCT system's software performs automatic segmentation of these vessel layers. The capillaries between the inner limiting membrane and the posterior border of the inner plexiform layer (IPL) form the superficial plexus, while the deep plexus form the capillary layer between the posterior border of the IPL and the posterior border of the outer plexiform layer. Quantitative data consisting of RVD for each microvascular layer were generated by the device software. RVD was measured at two concentric circular regions: The fovea-centered $1.0 \mathrm{~mm}$ radius area and the parafoveal region from the $1.0-3.0 \mathrm{~mm}$ radius area.

\section{Statistical Analysis}

All statistical analyses were performed using SPSS Statistics, Version 20.0 (IBM Corp.; Armonk, NY, USA). Mean, standard deviation, median, frequency, and ratio values were used in descriptive statistics of the data. For the analysis of independent quantitative data, The Mann-Whitney $U$ test was used. For all tests, a $p<0.05$ was defined as statistically significant.

\section{RESULTS}

Thirteen eyes of 8 patients (4 males, 4 females) with MacTel2 were included in the study. The mean age of the patients was $64.6 \pm 3.8$ years (61-73). Three eyes had subfoveal SNV secondary to MacTel2.

The mean superficial (foveal and parafoveal) and deep (foveal and parafoveal) RVD measurements in patients with proliferative and non-proliferative MacTel2 are shown in Table 1. There was a significant difference only in deep foveal RVD between proliferative and non-proliferative MacTel2 patients (Figure 1).

\section{DISCUSSION}

MacTel2 is a bilateral condition with typical changes in the macular capillary network with neurosensitive atrophy. Distinguishing features of the disease include hyporeflective intraretinal cavitation, abnormal vascular anastomosis, retinal pigment epithelial hyperplasia, foveal thinning, and progressive photoreceptor loss due to pathophysiological and biochemical changes primarily involving Müller cells (9). Eyes with MacTel2 are divided into non-proliferative and proliferative. The non- 
proliferative stage is characterized by foveal atrophy and telangiectasia, while the proliferative stage is characterized by SNV (1,2). OCT features of non-proliferative MacTel2 are IS/ OS disruption, hyporeflective areas in the inner retinal layers and pigment plaques (10). FA is the best imaging method for diagnosing MacTel2 by showing dilated perifoveal capillaries with leakage in the parafoveal temporal area as well as being able to show right-angled vessels and intraretinal and/or subretinal anastomoses (11). When present, SNV is characterized by visualization of early and late fluorescence leakage; however, the existence of SNV may be difficult to detect due to fluorescence leakage in the corresponding region from the abnormal retinal vasculature $(12,13)$. With the development of OCTA, non-invasive imaging of the retina and choroidal microvasculature has been possible without the use of exogenous intravenous dye injection. OCTA is capable of detecting abnormal microvasculature in the perifoveal region and correlating with leakage seen in FA images on OCTA images. in addition, it has the typical advantages of OCT imaging in cases such as the ability to diagnose MacTel 2 and monitor its progress, and thus helps to confirm the diagnosis. OCTA is a non-invasive method and offers the advantages over FA of being faster, less espensive, safer, and easily repeatable;
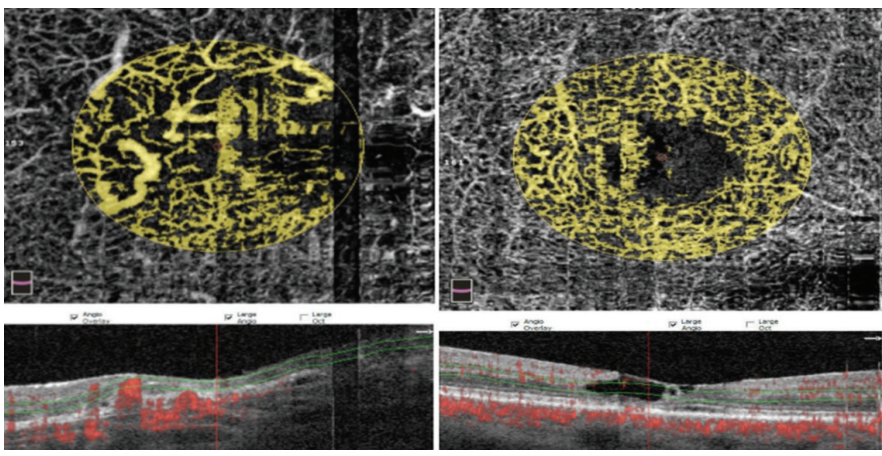

Figure 1. Deep retinal vascular density images provided with optical cohorence tomography angiography. Deep foveal retinal vascular density was significantly higher in proliferative MacTel2 (left) than in non-proliferative MacTel2 (right)

MacTel2: Macular telangiectasia type 2

Table 1. Retinal vascular density measurements in nonproliferative and proliferative MacTel2 patients

\begin{tabular}{|l|l|l|l|}
\hline & $\begin{array}{l}\text { Non-proliferative } \\
\text { MacTel2 }\end{array}$ & $\begin{array}{l}\text { Proliferative } \\
\text { MacTel2 }\end{array}$ & $\mathbf{p}$ \\
\hline $\begin{array}{l}\text { Foveal superficial } \\
\text { RVD }\end{array}$ & $30.3 \% \pm 9.3 \%$ & $39.4 \% \pm 3.3 \%$ & 0.31 \\
\hline $\begin{array}{l}\text { Parafoveal } \\
\text { superficial RVD }\end{array}$ & $49.5 \% \pm 6.4 \%$ & $43.8 \% \pm 2.9 \%$ & 0.41 \\
\hline Foveal deep RVD & $30.4 \% \pm 7.8 \%$ & $47.5 \% \pm 0.2 \%$ & $\mathbf{0 . 0 2}$ \\
\hline Parafoveal deep RVD & $50.7 \% \pm 3.8 \%$ & $55.3 \% \pm 8.4 \%$ & 0.23 \\
\hline RVD: Retinal vascular density, MacTel2: Macular telangiectasia type 2 \\
\hline
\end{tabular}

producing a better image quality; and generating fewer effects from fluorescent leaks that hide the microvasculature (5-7). Previous studies have demonstrated multiple telangiectatic, microaneurysm-like dilated vessels in the middle retinal layers on OCTA imaging. SNV, which is a neovascular complex, communicates with both the choroidal and retinal circulation, and OCTA imaging is capable of providing better visualization of SNV than FA due to the absence of fluorescence leakage. OCTA imaging is often useful for monitoring non-proliferative MacTel2 patients for timely detection of subretinal neovascular changes, as imaging can be repeated frequently (12-14). In this study, we found that deep foveal RVD was significantly higher in proliferative MacTel2 than in non-proliferative MacTel2. This may have been due to the hyporeflective intraretinal cavitation in non-proliferative MacTel2 and subfoveal vascular membrane in prolifertive MacTel2. While the features of SNV with OCTA have been described thoroughly in previous studies, the procurement of quantitative data on the deep retinal layers may help to differentiate proliferative MacTel2 from non-proliferative disease.

\section{Study Limitations}

The limitations of the current study are its retrospective design and the limited number of included patients. Further prospective studies with larger sample sizes are needed to confirm the realistic value of RVD in the diagnosis of SNV in MacTel2.

\section{CONCLUSION}

We obtained superficial and deep foveal and parafoveal RVD in patients with either non-proliferative or proliferative MacTel2. The results of this study showed that quantification of RVD in different retinal layers using OCTA imaging is a promising method for use in the diagnosis of SNV secondary to MacTel2.

\section{Ethics}

Ethics Committee Approval: İstanbul Beyoğlu Eye Training and Research Hospital Review Board (approval date: 31.08.2018, approval no: 18/l-1).

Informed Consent: Retrospective consent of the patient was not obtained, since measurements were not performed prospectively on the patient and previous documents were scanned.

Peer-review: Externally and internally peer-reviewed.

\section{Authorship Contributions}

Concept: A.D., Z.A., G.E., Design: A.D., Z.A., G.E., Data Collection or Processing: A.D., C.Y., G.G., D.Y., Analysis or Interpretation: A.D., C.Y., G.G., Literature Search: A.D., G.E., Writing: A.D. 
Conflict of Interest: No conflict of interest was declared by the authors.

Financial Disclosure: The authors declared that this study received no financial support.

\section{REFERENCES}

1. Gass JD, Blodi BA. Idiopathic juxtafoveolar retinal telangiectasis. Update of classification and follow-up study. Ophthalmology 1993;100:153646.

2. Yannuzzi LA, Bardal AM, Freund KB, Chen KJ, Eandi CM, Blodi B. Idiopathic macular telangiectasia. Arch Ophthalmol 2006;124:450-60.

3. Tan GS, Kuehlewein L, Sadda SR, Sarraf D, Schwartz SD. Subretinal neovascularization in macular telangiectasia type 2: optical coherence tomographic angiography and treatment response. Retin Cases Brief Rep 2015;9:286-9.

4. Gass JD, Oyakawa RT. Idiopathic juxtafoveolar retinal telangiectasis. Arch Ophthalmol 1982;100:769-80.

5. Roisman L, Rosenfeld PJ. Optical Coherence Tomography Angiography of Macular Telangiectasia Type 2. Dev Ophthalmol 2016;56:146-58.

6. Jia Y, Bailey ST, Wilson DJ, Tan O, Klein ML, Flaxel CJ, et al. Quantitative optical coherence tomography angiography of choroidal neovascularization in age-related macular degeneration. Ophthalmology 2014;121:1435-44.

7. Jia Y, Tan O, Tokayer J, Potsaid B, Wang Y, Liu JJ, et al. Split-spectrum amplitude-decorrelation angiography with optical coherence tomography. Opt Express 2012;20:4710-25.
8. Balaratnasingam C, Yannuzzi LA, Spaide RF. Possible choroidal neovascularization in macular telangiectasia type 2. Retina 2015;35:2317-22.

9. Nowilaty SR, Al-Shamsi HN, Al-Khars W. Idiopathic juxtafoveolar retinal telangiectasis: a current review. Middle East Afr J Ophthalmol 2010;17:224-41.

10. Paunescu LA, Ko TH, Duker JS, Chan A, Drexler W, Schuman JS, et al. Idiopathic juxtafoveal retinal telangiectasis: new findings by ultrahighresolution optical coherence tomography. Ophthalmology 2006;113:4857.

11. Soheilian M, Tavallali A, Peyman GA. Identification of intraretinal neovascularization by high-speed indocyanine green angiography in idiopathic perifoveal telangiectasia. Ophthalmic Surg Lasers Imaging 2007;38:167-9.

12. Thorell MR, Zhang Q, Huang Y, An L, Durbin MK, Laron M, et al. Sweptsource OCT angiography of macular telangiectasia type 2. Ophthalmic Surg Lasers Imaging Retina 2014;45:369-80.

13. Gonzalez MA, Shechtman D, Haynie JM, Semes L. Unveiling idiopathic macular telangiectasia: clinical applications of optical coherence tomography angiography. Eur J Ophthalmol 2017;27:e129-e33.

14. Toto L, Di Antonio L, Mastropasqua R, Mattei PA, Carpineto P, Borrelli E, et al. Multimodal Imaging of Macular Telangiectasia Type 2: Focus on Vascular Changes Using Optical Coherence Tomography Angiography. Invest Ophthalmol Vis Sci 2016;57:0CT268-76. 